\title{
Quantum Semantic Correlations in Hate and Non-Hate Speeches*
}

\author{
Francesco Galofaro \\ Politecnico \\ Milano, Italy \\ Free University of Bozen \\ 39100 Bozen, Italy \\ francesco.galofaro@polimi.it \\ Zeno Toffano Bich-Liên Doan \\ CentraleSupélec \\ Gif-sur-Yvette, France \\ zeno.toffano@centralesupelec.fr Bich-lien.doan@centralesupelec.fr
}

\begin{abstract}
This paper aims to apply the notions of quantum geometry and correlation to the typification of semantic relations between couples of keywords in different documents. In particular we analysed texts classified as hate / non hate speeches, containing the keywords women, white, and black. The paper compares this approach to cosine similarity a classical methodology, to cast light on the notion of "similar meaning".
\end{abstract}

\section{Corpus}

The Online Hate Index[1], a joint initiative of Anti Defamation LeaguefLs Center for Technology and Society and UC BerkeleyfLs D-Lab, consists of 7619 comments from the platform Reddit, collected in 2016 during the USA Presidential campaign. While the corpus has been funded by ADL, no research activities are conducted by ADL or directed based on their personal goals: research design and testing has been conducted entirely by Berkeley D-Lab, an interdisciplinary academic unit. The comments were labelled by a team of undergraduates under the supervision and using methods developed by D-Lab. ADL had no involvement in the selection of comments, development of methods, and labels applied to comments. 7184 comments have been manually labelled "non-hate", whereas 411 have been considered as "hate" speeches with the purpose of stimulating further machine learning researches. The five most used words in the collected hate speeches are Jews, white, hate, black, and women. Hate speeches present also some peculiar features: the average number of words, the average number of all caps, and the average sentence length is higher. Among the five most used words in hate speeches, white and black are interesting because they can be considered an antonymic couple from the point of view of lexical semantics. For this reason, we decided to analyse the semantic relations between the terms white, black, and women.

\subsection{Sub-corpora}

As the corpus is subdivided into hate (see table 1) and non-hate speeches (see table 2), we identified six sub-corpora:

${ }^{*}$ This paper has been made possible thanks to a corpus of hate and non-hate speeches shared by the Berkeley D-Lab.

B. Coecke, J. Hedges, D. Kartsaklis, M. Lewis, D. Marsden (Eds.):

2018 Workshop on Compositional Approaches

for Physics, NLP, and Social Sciences (CAPNS)

EPTCS 283, 2018, pp. 62-74 doi $10.4204 /$ EPTCS.283.5 
- H.WBWh: hate speeches corresponding to the logical expression black $*$ white $*$ women;

- H.WhW-B: white *women - black hate speeches;

- H.BW-Wh: black *women - white hate speeches;

- NH.WBWh: black $*$ white $*$ women non-hate speeches;

- NH.WhW-B: white $*$ women - black non-hate speeches;

- NH.BW-Wh: black *women - white non-hate speeches;

As we can see, selected hate-speeches tend to use the words white, black, and women in the same document, whereas not-hate speeches tend to use only white, women or black, women. It is sometimes difficult to understand the criteria that have been used for labelling the corpus. In particular, NH.WBWh.2 and NH.WhW-B.4, two documents labelled as non-hate, though avoiding offensive terms contain stereotypes on the relation between race and IQ. We hope that a semantic analysis will be helpful to re-discuss the classification in case of mistakes, e.g. if a document classified as non-hate speech shows the same semantic features of hate speeches.

\subsection{Note on the formalism}

The logical formalism we used to represent elementary semantic relations corresponds both to BooleFLs algebra and to the one proposed by Greimas and Rastier in their well-known semiotic square [9] and suggests a strong connection to Dubois and ToffanoFls eigenlogic which could provide a formal description of the semantic relations immanent to the text, thus allowing the formulation of semantic queries[6].

Table 1: Hate speeches. Italic underlines the occurences of the keywords.

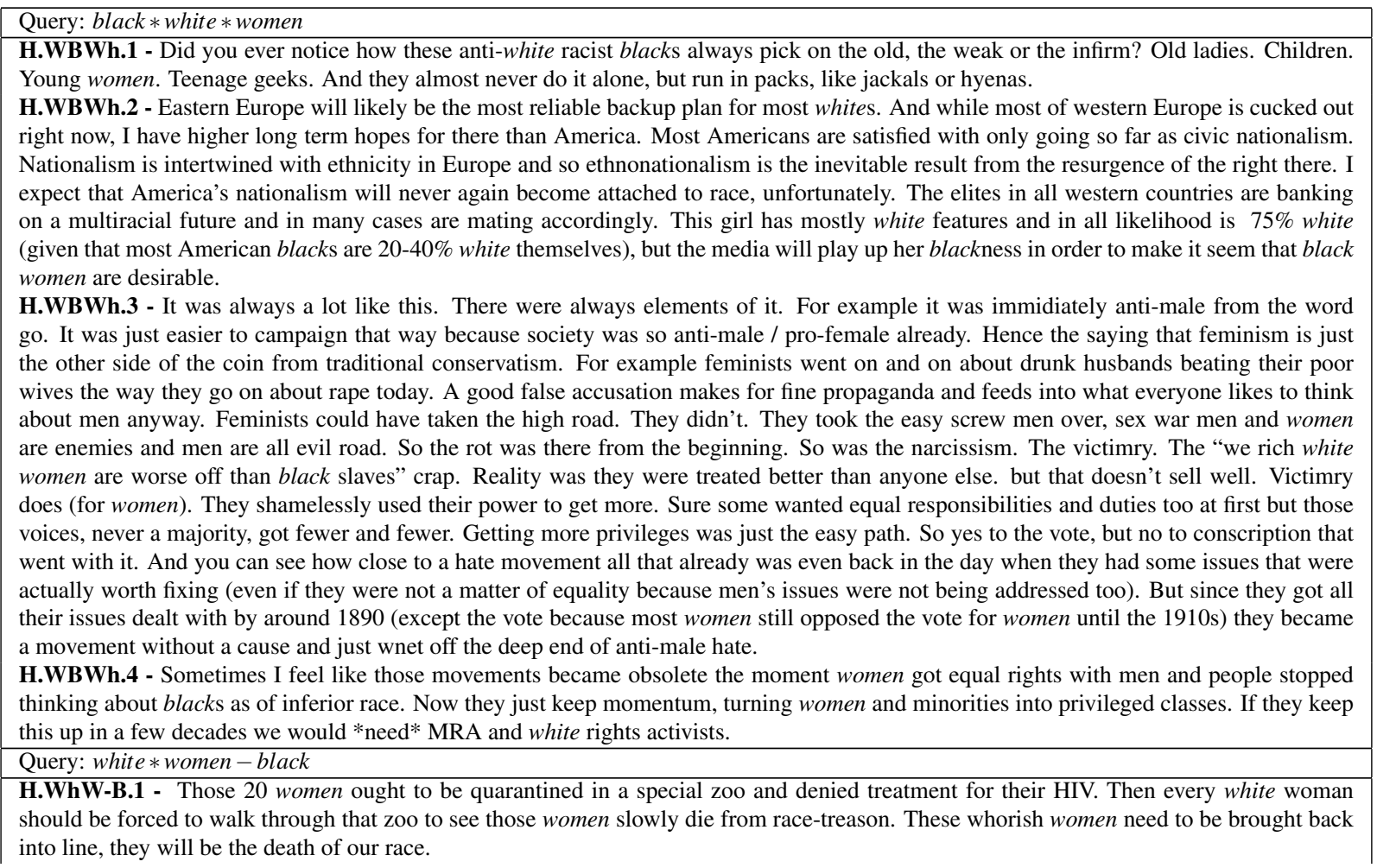


H.WhW-B.2 - Jewish gangs kidnapping/raping/killing white children as young as 2 years old to sell snuff films](http://www.jewwatch.com/jew-atrocities-blood-libel.html), then Jews had the Italian news executives fired for AIRING THE STORY (Jews HATE being called out on crime and DEMAND FULL CONCEALMENT or else it's "ANTI SEMITIC!!!"....Jews think it's ANTI SEMITIC to PROTECT YOUR CHILDREN FROM THEM). [Israel's \$1 billion a year sex slave trade of white women](http://www.jewlicious.com/2006/04/because-we-were-slaves-israels-sex-trade/)

H.WhW-B.3 There is no need to side with kikes against white women who put their money where their mouth is and goes and protests the jew at her own risk. Why on earth do I care if the Jews are weakening someone who is an avowed enemy, like Hamas? Granted they're not capable of it, but they've made declarations of intent for conquering Italy and other places in Europe. The Muslims will forever wage their jihad against Europe regardless of whether or not Israel exists. Their religion is one of rape and plunder. I don't really protest Israel because I couldn't care less what Israel does in Israel. If the time comes for Europe to reclaim Turkey and the Levant, then I will care. I care what Israeli stooges do here in the west, what propaganda they sow, what legislation they lobby for, etc.

Query: black*women - white

H.BW-Wh.1 - Based on the many, many videos I've watched of chimpouts, black women are more aggressive and more violent than black men. They seem to think there are no consequences for them when they punch other people in the face.

H.BW-Wh.2 - Liberals only teach the bad in american history. I had multiple teachers that told me that slavery affects black people today and women only make 70 cents to a man. These are both lies, and there is nothing taught about how we spread ideas of individual freedom across the western world and gave more rights to women, minorities, plants and animals than any other, all thanks to "racist slave holders" so yeah, teach slavery all you want, but also include the fact that these ideas were not constitutional and mostly pushed by democrats.

Table 2: Non-hate speeches. Italic underlines the occurrences of the keywords.

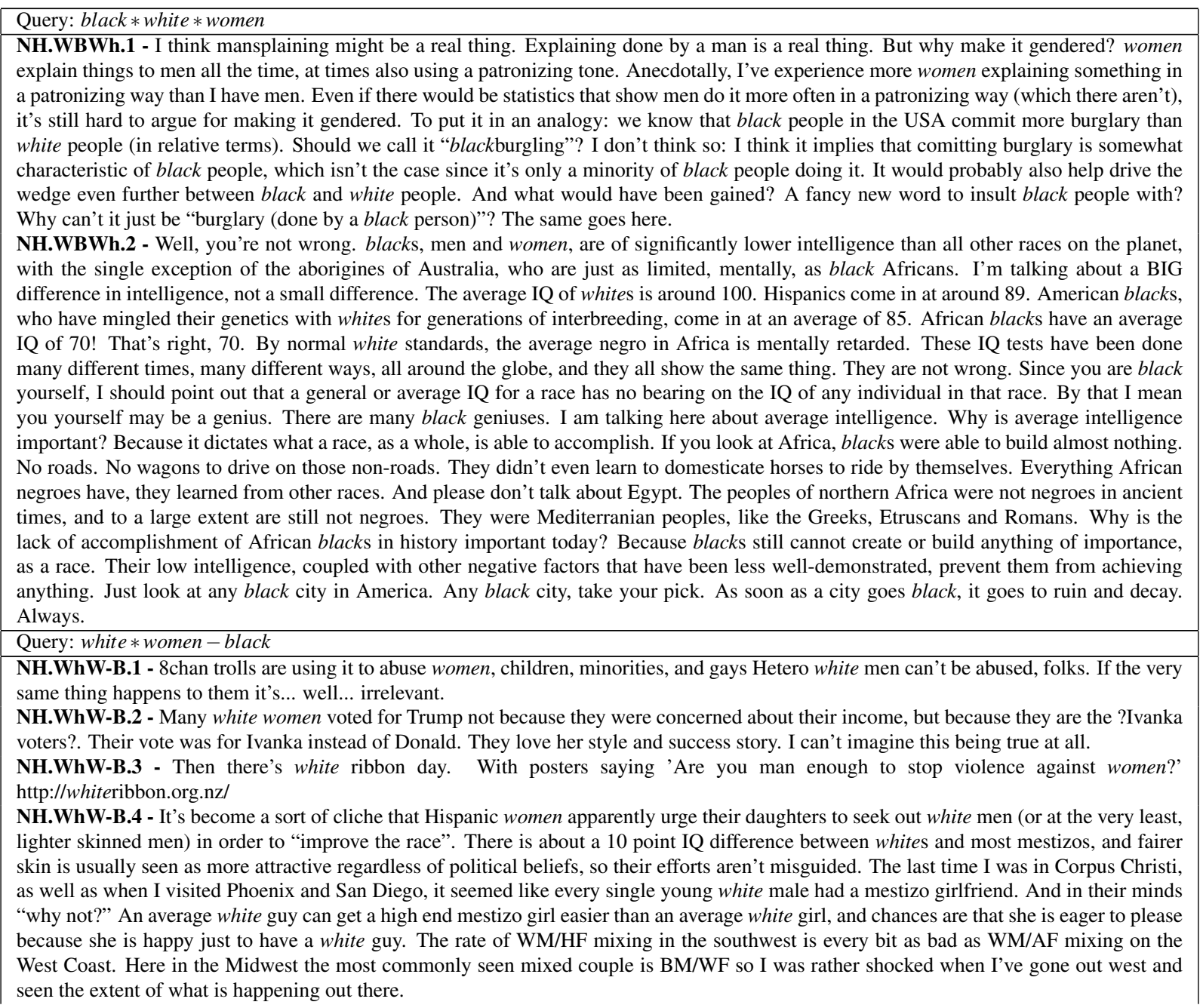


NH.WhW-B.5 - How would energising the base help. He already has them. "You would be in jail" "hate in your heart" and the "devil" comment are all for the base and he still dropped in the polls afterwards. The guy needs greater support from women, he needs greater support from minorities. As far as I know registration hasnt surged for the non college educated white voters so he needs that as well. Currently clinton can run out the clock, she won't and isn't trying that but she could rather comfortably not do much and let trump focus on attacking his accusers. I have a feeling he is going to leave that arguement behind now given that he has called the accusers horrible horrible liars and ?Take a look. Look at her. Look at her words. And you tell me what you think. I don?t think so,?

NH.WhW-B.6 - I wonder what she would say to me. I'm a white male that voted for Jill Stein/Ajamu Baraka. I voted for 2 women, rather than her 1.

Query: black *women - white

NH.BW-Wh.1 - Win or lose, a lot of people have got the red pill. Trump just did not play the demographics well. Alienating blacks, mexicans, muslims, women. But many of them also know what is happening, but they consider trump worse The establishment has suffered from a huge loss in credibility which they can never recover. As an example see the comments on cnn, abc, nyt facebook pages

NH.BW-Wh.2 - Unlike jubbergun, i didn't even notice the race of the attackers. I think one of the men standing around was black, but that was the only time i noticed color. Jubbergun is either a troll or a PC moron, that's all i can say. As for my specifically using bonobos rather than common chimps or gorillas, that is because the bonobos are our closest relatives and also one of the few species of primate amongst which the females commit most of the violence. Hence, these women have unleashed their inner bonobo.

NH.BW-Wh.3 - I'll admit, I'm really pro sanders but this upset me. He was pretty rude to the woman, I wish he let her talk more and had more of a conversation. To me, most the arguments I get into, the one who rages first is often the one with at least the most to lose and at most the least willed debater. Not to mention it is prejudice. Urban? Come on. If humans fight, if we're in a war, we use guns. Plain and simple. The black men in this country and black women in particular have been SHUT OUT of so many opportunities in this country and MANIPULATED into the war they think they have to wage. Manipulated by terrible, evil motherfuckers in badges who go around thinking they're doing the lord's work. And really, really fucking unfortunately, I can't help but feel that Sanders' view as the socialist democrat is so far right and so agreed upon by so many... it really does show how ingrained gun culture is in our country.

NH.BW-Wh.4 - claims quoting Dr. Martin Luther King, Jr., to black women is a violent and ?cisheteropatriarchy? act. You seriously can't make this up.

NH.BW-Wh.5 - That's probably because 30 years ago they were not bashing blacks or women. Well, women only got bashed if they mouthed off.

\section{State-of-the-art}

A geometric approach to semantic space studies has been proposed first by Jean Petitot, in terms of catastrophe theory[14]. As quantum geometry is concerned, it is used by scholars in Information Retrieval for the purpose of unifying vector, logic, and statistical approaches[19, 12]. Among others, Bruza and Woods [3], applied it to the semantic representation of polysemic words as a superposition state. Barros, Toffano, Meguebli and Doan[2] proposed to interpret the notion of entanglement as a measure of the strength of the semantic relation between two query-words, both present in a certain document. To this purpose, using the Hyperspace Analogue to Language (HAL) method[11], the authors formalised the semantic space of a document as a square matrix, as we will explain hereafter. Many quantum information retrieval scholars prefer this technique because it is Hermitian and it allows the implementation of a density matrix [19, 12]. Instead of measuring cosine similarity between two keywords, the work in [2] makes use of the Gram-Schmidt orthogonalisation method to measure the degree of correlation between the words, characterized by the violation of a CSHS inequality [4]. Pushing forward this idea, Galofaro, Toffano, and Doan[8] proposed a theoretical paper in which observables are interpreted as semantic features. The Born rule is used to find the expectation values associated to the application of a specific observable to two word-vectors in order to measure the degree of correlation/anticorrelation between them[18]. The present paper aims to test this method, and to compare it with the classical cosine similarity measure.

\section{Relevance to language processing}

It is possible to ask how are we going to interpret the correlation value in terms of linguistic features. According to Umberto Eco[7], the terms "semantics" has been used in five different acceptations: 
1. Lexicology: a study of meaning outside every context (dictionary);

2. Structural Semantics: interested in semantic fields considered as systems;

3. Study of the relation between the meaning and the referent;

4. Truth-conditional logic;

5. Textual semantics: a study of the peculiar meaning assumed by terms and words in their context;

Though the five levels are obviously related, the text and the context have the last word in defining the meaning of terms. For example, according to any thesaurus, black and white are antonyms (if black, then not white and vice versa). Having a look at our corpus, we find: "most American blacks are 20$40 \%$ white" (H.WBWh.2), weakening the antonymy. The HAL method allows us to work on semantics in sense of 5 because we formalise the semantic relations between terms that constitute a given context. These become the characteristics of a semantic space.

\subsection{Commutation test and quantum correlation}

With measuring the degree of quantum correlation we are searching for a semantic equivalent to Hjelmslevfl commutation test. Commutation of elements of the expression plan aims to search for linguistic units. If we substitute "black" with "Afro-American" in blacks, men and women are of significantly lower intelligence than all other races on the planet, we notice how meaning is unaltered, while if we substitute it with "Afghan hound", the meaning changes. We could even suggest that this is why the original sentence is actually racist: we speak about men as they were dogs. However, *Afghan hounds, men and women is not correct in English because of structural reasons related to semantics in sense 2: "men" and "women" carry a structural classeme (an element of meaning) (human $\rightarrow$-animal)[10] . What if we were able to commute meanings, and not signifiers? For example, what if we were able to change the meaning unit "human" in "dog" while preserving "male" and "female" all along the sentence? This is what we mean by "commutation test on the content plan". As a result of the test, such an abstract machine as a computer could probably generate, on the expression plan, a sentence like Afghan hounds, sires and bitches. The Born rule provides a tool to measure the expectation values for these commutations.

\section{Design}

In synthesis, we prepared the corpus reducing each word to its stem; we then applied the HAL method to obtain two word vectors representing the keywords we are interested in, and a document vector; finally, we measured the the cosine similarity of the keywords and their (anti)correlation value.

\subsection{Cleaning the corpus}

Since we are interested in every kind of semantic information not manifested by morphology or syntax, we used the Python library nltk Lancaster stem to reduce different tokens to the same type (e.g. black, blacks, blackness). The Lancaster stemmer is more aggressive than the alternative nltk Porter stemmer, which can distinguish between woman and women. Obviously, a stem is not necessarily identical to its morphological root: our purpose is only to reconstruct the immanent net of relations underlying the manifest words. For a similar reason, we used ntlk stopwords list to eliminate syncategorematic terms. We also used regex to eliminate all not relevant signs such as punctuation[20]. 


\subsection{The matrix}

We applied the HAL method to each document of each sub-corpus to formalise it. Given $k$ roots occurring in the document, we calculate a $k \times k$ matrix which represents the semantic space of the document. For example, table 3 shows the HAL matrix of the document:

NH.BW-Wh.5: That's probably because 30 years ago they were not bashing blacks or women. Well, women only got bashed if they mouthed off

Table 3: HAL Matrix corresponding to document NH.BW-Wh.5 (window: 11)

\begin{tabular}{|c|c|c|c|c|c|c|c|c|c|c|c|c|c|c|c|c|c|c|c|c|}
\hline & 30 & ago & bash & becaus & black & got & if & mouth & not & off & onli & or & probabl & $\mathbf{s}$ & that & they & well & were & women & year \\
\hline 30 & 10 & 0 & 0 & 9 & 0 & 0 & 0 & 0 & 0 & 0 & 0 & 0 & 8 & 7 & 6 & 0 & 0 & 0 & 0 & 0 \\
\hline ago & 8 & 10 & 0 & 7 & 0 & 0 & 0 & 0 & 0 & 0 & 0 & 0 & 6 & 5 & 4 & 0 & 0 & 0 & 0 & 9 \\
\hline bash & 4 & 6 & 22 & 3 & 3 & 9 & 0 & 0 & 10 & 0 & 8 & 4 & 2 & 1 & 0 & 7 & 6 & 8 & 12 & 5 \\
\hline becaus & 0 & 0 & 0 & 10 & 0 & 0 & 0 & 0 & 0 & 0 & 0 & 0 & 9 & 8 & 7 & 0 & 0 & 0 & 0 & 0 \\
\hline black & 3 & 5 & 9 & 2 & 10 & 0 & 0 & 0 & 8 & 0 & 0 & 0 & 1 & 0 & 0 & 6 & 0 & 7 & 0 & 4 \\
\hline got & 0 & 0 & 3 & 0 & 4 & 10 & 0 & 0 & 2 & 0 & 9 & 5 & 0 & 0 & 0 & 0 & 7 & 1 & 14 & 0 \\
\hline if & 0 & 0 & 10 & 0 & 2 & 8 & 10 & 0 & 0 & 0 & 7 & 3 & 0 & 0 & 0 & 0 & 5 & 0 & 10 & 0 \\
\hline mouth & 0 & 0 & 7 & 0 & 0 & 6 & 8 & 10 & 0 & 0 & 5 & 1 & 0 & 0 & 0 & 9 & 3 & 0 & 6 & 0 \\
\hline not & 5 & 7 & 0 & 4 & 0 & 0 & 0 & 0 & 10 & 0 & 0 & 0 & 3 & 2 & 1 & 8 & 0 & 9 & 0 & 6 \\
\hline off & 0 & 0 & 6 & 0 & 0 & 5 & 7 & 9 & 0 & 10 & 4 & 0 & 0 & 0 & 0 & 8 & 2 & 0 & 4 & 0 \\
\hline onli & 0 & 0 & 4 & 0 & 5 & 0 & 0 & 0 & 3 & 0 & 10 & 6 & 0 & 0 & 0 & 1 & 8 & 2 & 16 & 0 \\
\hline or & 2 & 4 & 8 & 1 & 9 & 0 & 0 & 0 & 7 & 0 & 0 & 10 & 0 & 0 & 0 & 5 & 0 & 6 & 0 & 3 \\
\hline probabl & 0 & 0 & 0 & 0 & 0 & 0 & 0 & 0 & 0 & 0 & 0 & 0 & 10 & 9 & 8 & 0 & 0 & 0 & 0 & 0 \\
\hline $\mathbf{s}$ & 0 & 0 & 0 & 0 & 0 & 0 & 0 & 0 & 0 & 0 & 0 & 0 & 0 & 10 & 9 & 0 & 0 & 0 & 0 & 0 \\
\hline that & 0 & 0 & 0 & 0 & 0 & 0 & 0 & 0 & 0 & 0 & 0 & 0 & 0 & 0 & 10 & 0 & 0 & 0 & 0 & 0 \\
\hline they & 7 & 9 & 8 & 6 & 1 & 7 & 9 & 0 & 0 & 0 & 6 & 2 & 5 & 4 & 3 & 20 & 4 & 0 & 8 & 8 \\
\hline well & 0 & 2 & 6 & 0 & 7 & 0 & 0 & 0 & 5 & 0 & 0 & 8 & 0 & 0 & 0 & 3 & 10 & 4 & 9 & 1 \\
\hline were & 6 & 8 & 0 & 5 & 0 & 0 & 0 & 0 & 0 & 0 & 0 & 0 & 4 & 3 & 2 & 9 & 0 & 10 & 0 & 7 \\
\hline women & 1 & 4 & 12 & 0 & 14 & 0 & 0 & 0 & 10 & 0 & 0 & 16 & 0 & 0 & 0 & 6 & 9 & 8 & 28 & 2 \\
\hline year & 9 & 0 & 0 & 8 & 0 & 0 & 0 & 0 & 0 & 0 & 0 & 0 & 7 & 6 & 5 & 0 & 0 & 0 & 0 & 10 \\
\hline
\end{tabular}

Each square matrix is calculated moving a window, representing the considered context, over the document, stem by stem. All stems within the window co-occur with the last stem with a strength which is inversely proportional to the distance between the stems. We finally sum the different occurrences of stems: for example, women occurs two times in our document.

\subsection{Cosine similarity}

In a HAL matrix, rows and columns differ. A word-vector is then represented by the concatenation between the corresponding row and column vectors. In this way we obtain the word-vectors of the keywords we are interested in (white, black, women): $\left|w_{\text {white }}\right\rangle,\left|w_{\text {black }}\right\rangle,\left|w_{\text {women }}\right\rangle$. We can now calculate the angle between any two word-vectors as well as their cosine similarity (cs), since "cosine has the nice property that it is 1.0 for identical vectors and 0.0 for orthogonal vectors" [16]. Usually, cosine similarity measures the similarity between the query vector and the document vector. For this reason, the way we use it, measuring cosine similarity between the keywords in each document, and obtaining each time a different measure, could seem rather unorthodox. However, since the two keywords are just vectors, their angle can be used to measure their similarity in the particular semantic space corresponding to a certain document. For example, as Song, Bruza, and Cole wrote, "nurse and doctor are similar in semantics to each other, as they always experience the same contexts, i.e., hospital, patients, etc.'[17]. The reason why we choose to compare cosine similarity to the expectation degree measured through the Born rule is perhaps not intuitive. In both case we deal with many-dimensional vectors, and not with punctiform 
events. For this reason we will not consider the euclidean distance to calculate similarity or different methods to calculate frequency, such as pointwise mutual information (PPMI).

\subsection{Gram-Schmidt orthogonalisation}

In order to measure correlation between two keyword-vectors, let us say black and women, we first obtain a document vector $|\Psi\rangle$ summing all word-vectors. The next step is to apply the Gram-Schmidt orthogonalisation process to $\left|w_{\text {black }}\right\rangle,\left|w_{\text {women }}\right\rangle$ in order to obtain two pairs of orthonormal bases $\left\{\left|u_{\text {black }}\right\rangle,\left|u_{\text {black } \perp}\right\rangle\right\}$ and $\left\{\left|u_{\text {women }}\right\rangle,\left|u_{\text {women } \perp}\right\rangle\right\}$. If we project and normalise the document-vector $|\Psi\rangle$ onto each couple of bases we obtain a vector $|\phi\rangle$ :

$$
|\phi\rangle=\alpha\left|u_{\text {black }}\right\rangle+\alpha_{\perp}\left|u_{\text {black } \perp}\right\rangle=\beta\left|u_{\text {women }}\right\rangle+\beta_{\perp}\left|u_{\text {women } \perp}\right\rangle
$$

We want to emphasize that we represented the document vector through its components on the two bases provided by each keyword-vector.

\subsection{Abstract machines}

The notion of abstract machine links quantum theory [18] to post-structuralist perspectives on meaning [5]. To typify the semantic relation between black and women in our corpus, we design two abstract machines: $\sigma$ and $\tau$, two linear operators. Their input vector is $|\phi\rangle$ (representing the document. The $\sigma$-machine operates on each context, and returns the output +1 when it acts on the vector $\left|u_{\text {black }}\right\rangle$ (representing the meaning of the stem black), -1 in the other case. In a similar way, the $\tau$-machine applies the same transformation on the meaning of the stem women. Now let us imagine what happens when we apply both the machines to the document: $\sigma \tau|\phi\rangle$. Principally, we deal with three situations:

1. the two outcomes are correlated in every context, when the first output is +1 and the second is +1 , and when the first is -1 , the second is -1 . If we multiply the two numbers we always score +1 ;

2. the two outcomes are anti-correlated: in every context where the first output is +1 , the second will be -1 and also the other way round. If we multiply the two numbers, we will always score -1 ;

3. the two outcomes are not correlated. in some contexts the output of the two machines will be $\{+1,+1\}$, while in others it will be $\{+1,-1\},\{-1,+1\}$, or $\{-1,-1\}$. The average of the outcomes in the different contexts of the considered document will be 0 ;

The three considered cases are extreme situations: we will also find weak correlations, in which the score will tend to 1 , weak anti-correlation, where the score will tend to -1 , and also absence of correlation, giving results near 0 . The outcome of a generic machine can be a transformation or not, + or - . Since we have two machines, we deal with a four-state semantic space $\sigma \tau=\{++,+-,-+,--\}$. To construct an example of a general machine, we use the following Pauli spin matrix:

$$
\hat{\sigma}_{x}=\left(\begin{array}{ll}
0 & 1 \\
1 & 0
\end{array}\right)
$$

The effect of this matrix is to switch the components of the state-vector to which it is applied. It is the equivalent of the logical gate negation in Quantum Computation[13]. In this way we define an operator $\hat{B}_{x}$ in the black-base $\left\{\left|u_{\text {black }}\right\rangle,\left|u_{\text {black } \perp}\right\rangle\right\}$, and an operator $\hat{W}_{x}$ in the women-base $\left\{\left|u_{\text {women }}\right\rangle,\left|u_{\text {women } \perp}\right\rangle\right\}$. As a result, for example, $\hat{W}_{x}$ switches all the $\left|u_{\text {women }}\right\rangle$-related components of $|\phi\rangle$ :

$$
\hat{W}_{x}\left(\beta\left|u_{\text {women }}\right\rangle+\beta_{\perp}\left|u_{\text {women } \perp}\right\rangle\right)=\beta \perp\left|u_{\text {women }}\right\rangle+\beta\left|u_{\text {women } \perp}\right\rangle
$$


$\hat{B}_{x}$ acts in the same manner on the $\left|u_{\text {black }}\right\rangle$-related values of $|\phi\rangle$. To calculate the expected score of the application of both machines to the document-vector, we apply the Born rule, whose output will be a number between -1 and 1 .

$$
r=\left\langle\phi\left|\hat{B}_{x} \hat{W}_{x}\right| \phi\right\rangle
$$

The more the two layers of meaning are connected, the more the two independent abstract machines will return similar outputs: thus we interpret $r$ as the immanent correlation between the respective meanings expressed by the black and the women stems.

\section{Comparing data}

We measured cosine similarity and correlation in our corpus of hate and non-hate speeches corresponding to the logical query black*white*women. We measured similarity and correlation between black, women and white, women respectively. The window length varies from 4 to 10 . The results are displayed in fig. 1. referring to hate speeches, and fig. 2 referring to non-hate speeches. Then we calculated the graphs corresponding to cosine similarity and correlation between white, women in the corpora of both hate and non-hate speeches where the term black is absent - see figures 3 and 4 . Finally, we applied the same procedure to black, women in the corpora of both hate and non-hate speeches where the term white is absent - see figures 5 and 6 . To compare the results we focus on window lengths of 8-10, which seem associated to more stable values.

\section{1 black $*$ white $*$ women}

Both hate and non-hate speeches present strong anticorrelation black vs. women and white vs. women. Looking at the document H.BWhW.4, it is not surprising to see that both black/women and white/women are anticorrelated, since the text draws a comparison between women's rights and black's rights. We notice also the correspondence between a $r \simeq 0$ correlation score and a $c s \simeq 0.7$ value of similarity: two word-vectors can be geometrically close without being correlated. Another interesting problem is the black/white opposition. Generally speaking, their anticorrelation is weaker than for the other two, and it tends to disappear in H.WBWh.1 Provided that lexical semantics would describe them as antonyms, it could seem strange that they are not anti-correlated in H.WBWh.1. According to textual semantics [10] semantic relations are modulated and transformed by their co-occurrence in contexts. In our document, anti-, white, and racist give a fundamental contribution to establish the contextual part of the meaning of black, providing it of contextual classemic values - Rastier calls them afferent semes, and distinguishes them from the inherent semes, which characterize the semantic nucleus of a lexeme[15]. In a similar way, H.WBWh.3 is also interesting, since it shows how two terms can be weakly similar $(0.5 \leq c s \leq 0.7)$ and still weakly anticorrelated $(-0.5 \leq r \leq 0)$.

\section{2 white $*$ women - black}

Five out of six non-hate speeches present a strong anticorrelation white vs. women, whereas hate speeches are featured by a weaker anticorrelation; in one case, NH.WhW-B.2, we have a positive correlation, since the document focuses on white women voting for D. Trump. If we look at the other documents, they oppose women to white men, voters. We must point out how NH.WhW-B.4 could be considered a hate speech from a semantic point of view. In this text, hispanic women are opposed to white girl, men, guys and this explains the strong anticorrelation. 

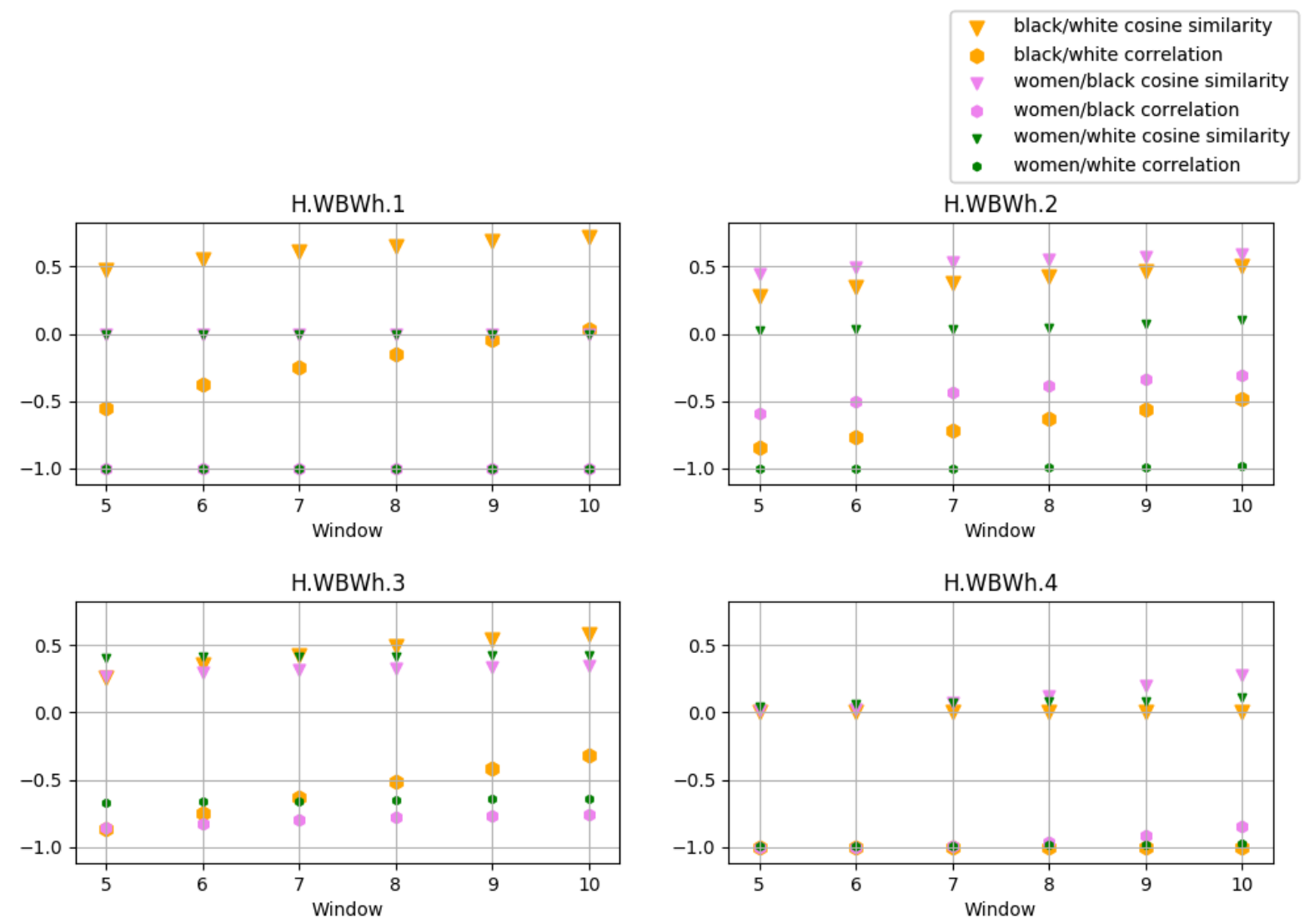

Figure 1: Hate speeches H.WBWh.1-4. In H.WBWh.1, black and white show a high similarity score though they are not correlated. In the text, the meaning of "white" is modified both by the prefix antiand by the presence of black.

NH.WBWh.1

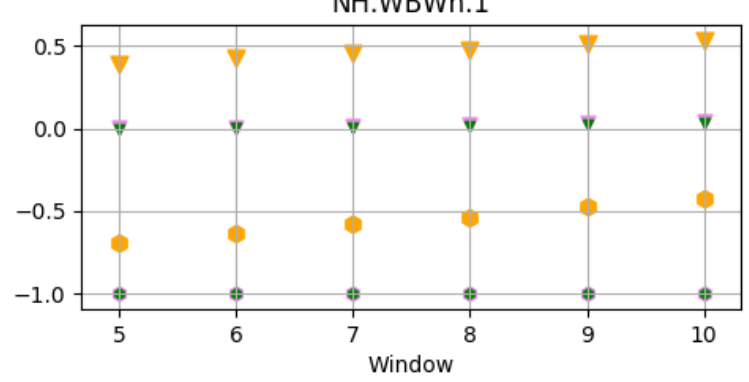

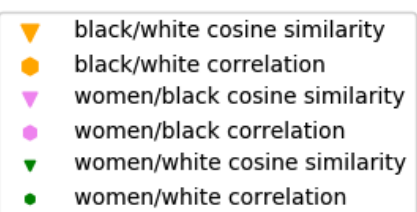

NH.WBWh.2

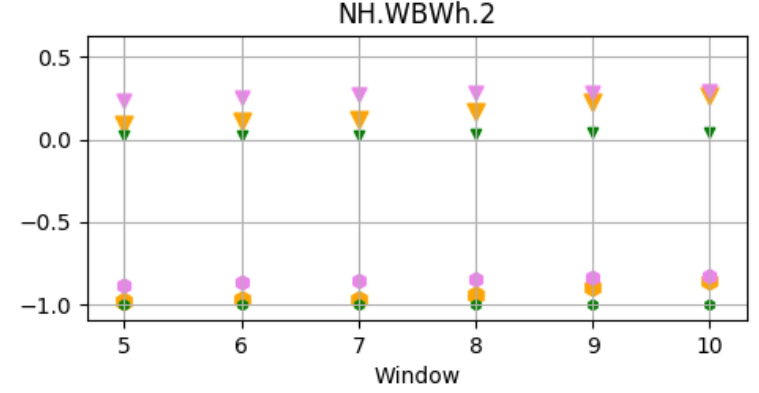

Figure 2: Non-hate speeches NH.WBWh.1-2. In NH.WBWh.1, a 0.5 similarity score between black and white corresponds to a -0.5 value of anticorrelation, since the text is between black and women. NH.WBWh.1 - a pseudo-scientific argument on IQ, opposes black and white 

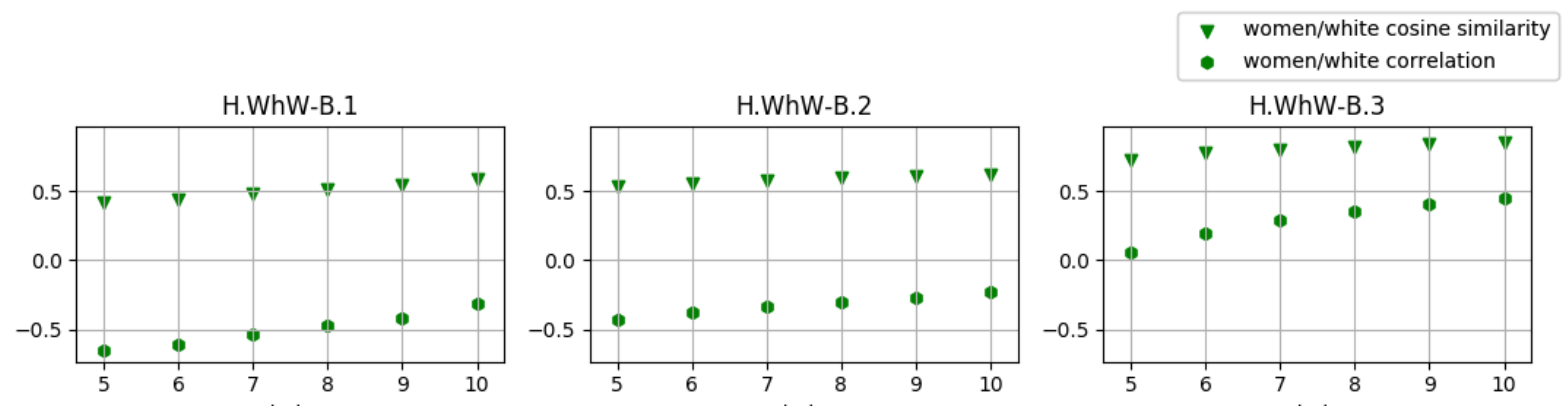

Figure 3: Hate speeches H.WhW-B.1-3. In particular, in H.WhW-B.1-2 the keywords occur without a strong relation, whereas H.WhW-B.3 is explicitly on white women
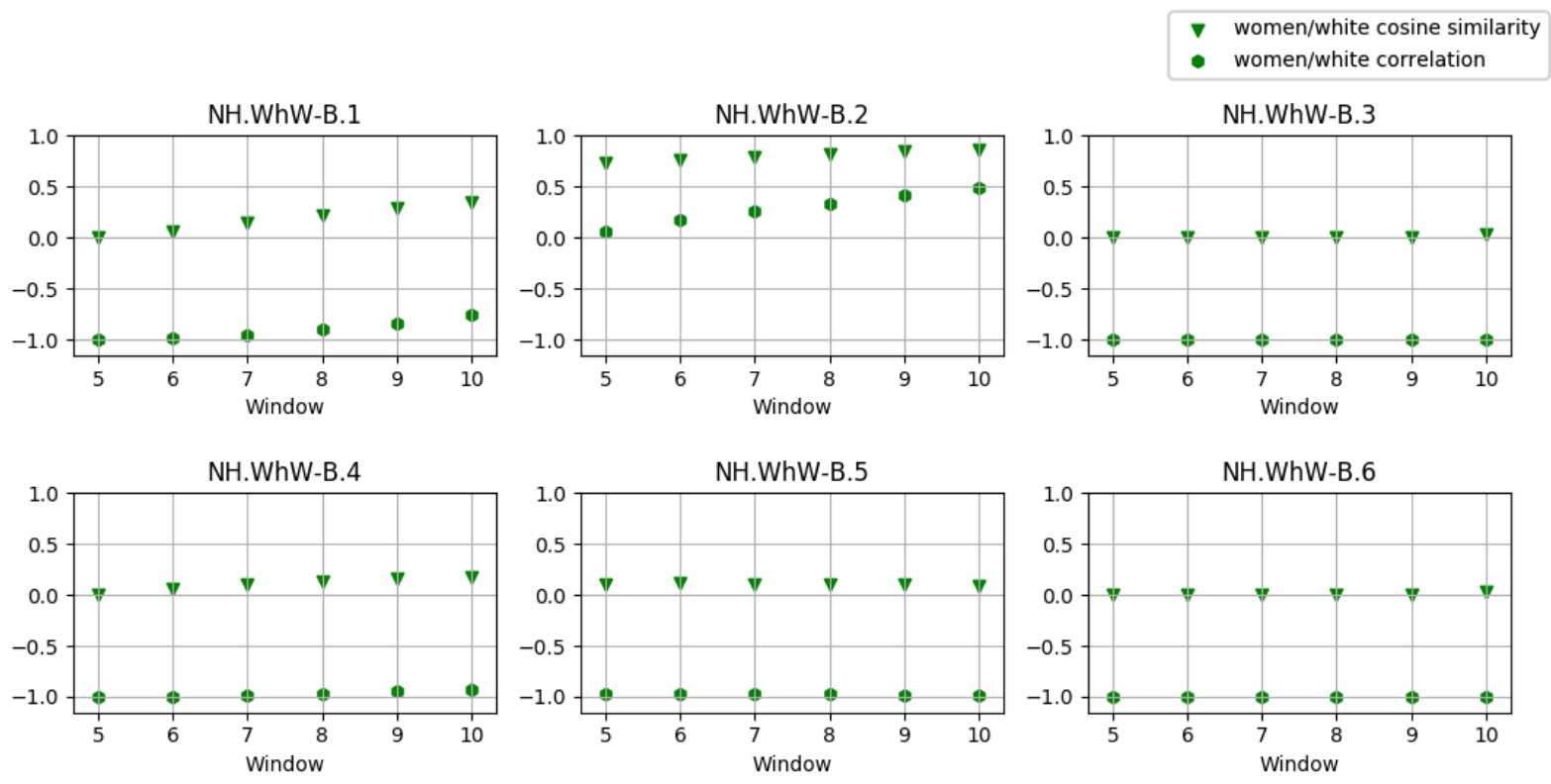

Figure 4: Non-hate speeches NH.WhW-B.1-6. Five out of six documents show a maximal anticorrelation and a 0 similarity score. NH.WhW-B.2 is about white women ("Ivanka Voters").

\section{3 black $*$ women - white}

H.WB-Wh.1 presents a positive correlation between black and women: in fact the document opposes black women to black men without reference to white women (women $\rightarrow$ black). On the contrary, H.WB-Wh. 2 present a strong anticorrelation black vs. women, since women and blacks are considered as two distinct minorities. Most non-hate speeches present a weak anticorrelation or a weak correlation, except for NH.WB-Wh.2, in which a maximal anticorrelation value is justified because the document is composed of two different sections, the first about black color and the second about women. In NH.BWWh.5 we can see again how a high score of similarity does not necessarily correspond to a correlation of sa given type: in this text, we have a first close co-occurrence of women and black; the second occurrence of women is free and it weakens the value of the first relation. 
$\nabla$ women/black cosine similarity

- women/black correlation
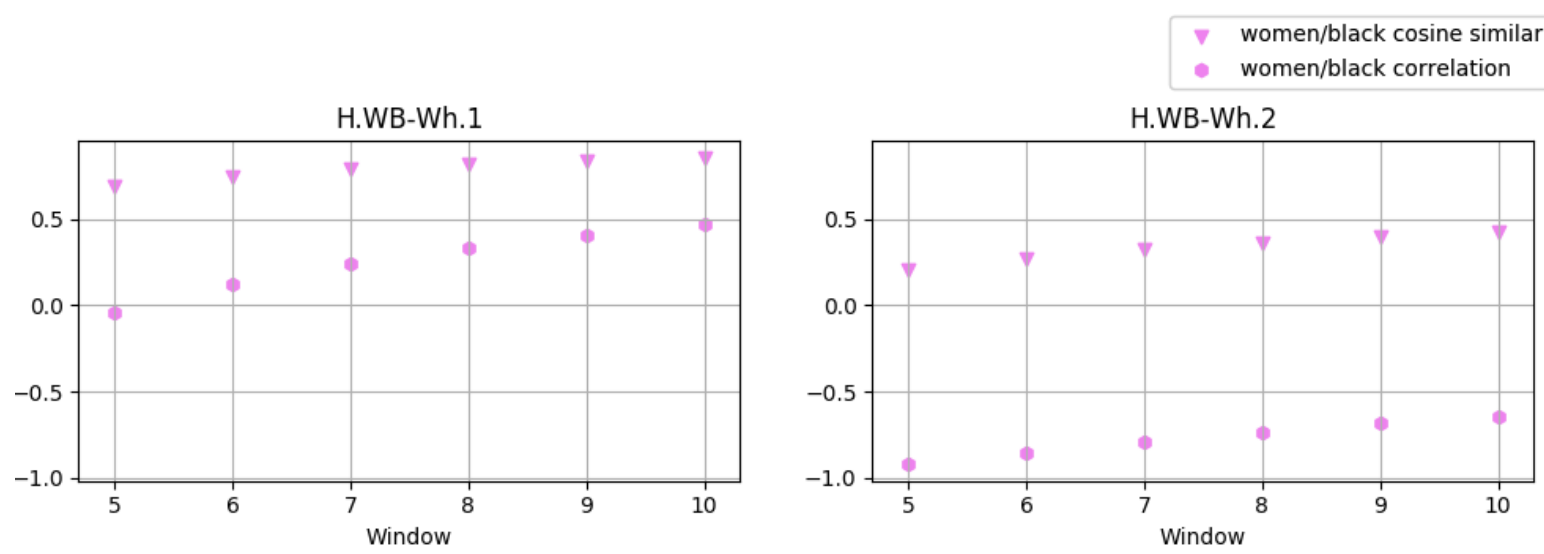

Figure 5: Hate speeches H.WB-Wh.1-2. H.WB-Wh.1 is about black women, opposed to black men; H.WB-Wh.2 carries on an analogy between blacks' rights and women's rights
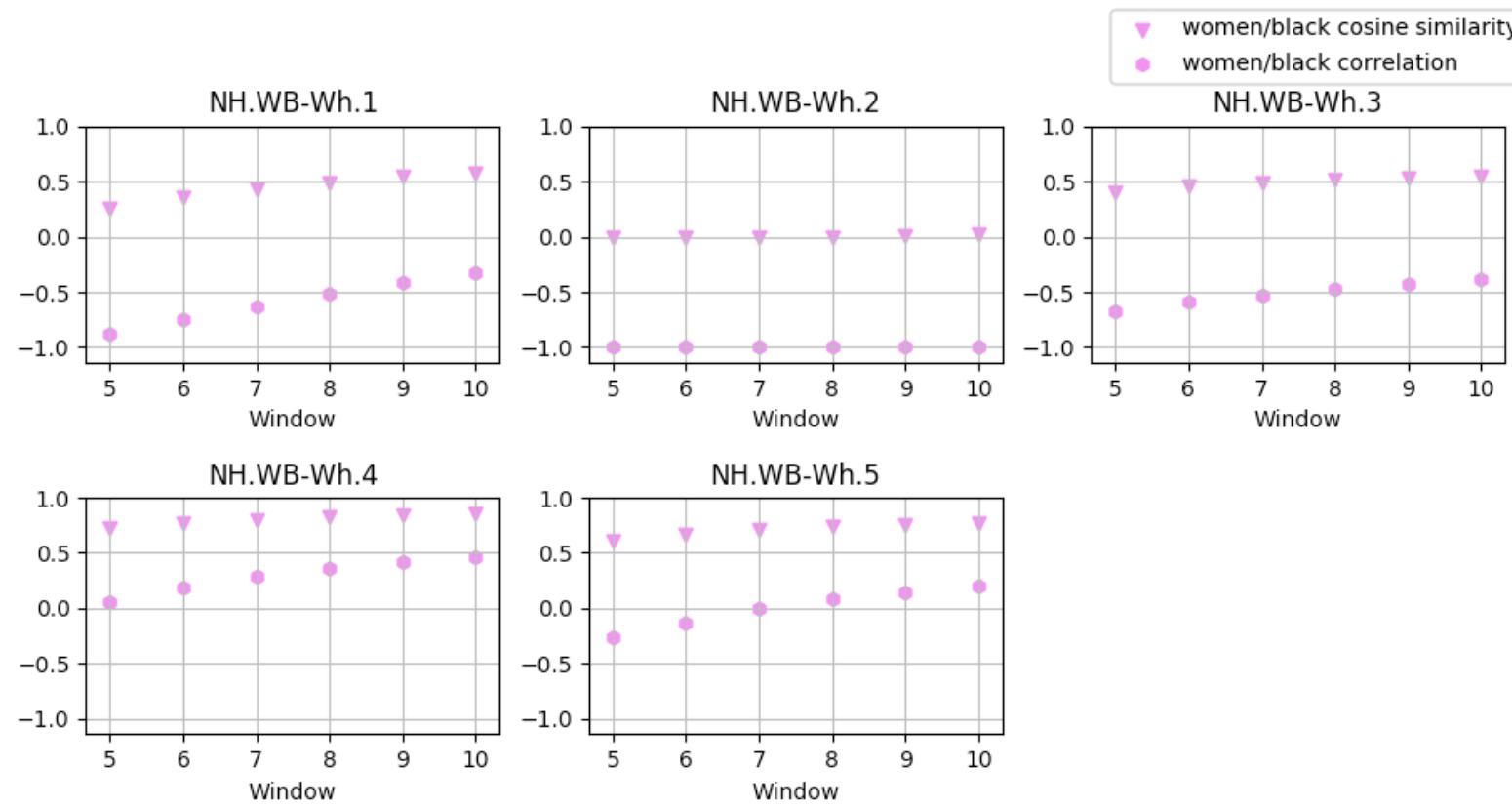

Figure 6: Non-hate speeches NH.WB-Wh.1-5 show a great differentiation. In these cases, correlation is helpful to characterise more explicitly what does "similarity" mean 


\section{Conclusion}

The paper shows how quantum correlation can clarify the less clear notion of similarity. The terms black and women can occur in hate-speeches with different relations, individuating black women, or opposing women and blacks. In particular:

- Low similarity values $(0 \leq c s \leq 0.5)$ correspond to a maximum of anticorrelation $(-1 \leq r \leq-0.5)$ between the two stems. We have a double privative semantic opposition $-(A * B)$;

- Weak similarity $(0.5 \leq c s \leq 0.7)$ correspond to weak anticorrelation or no correlation $(-0.5 \leq r \leq$ 0 );

- Higher similarity value $(0.7<c s \leq 1)$ corresponds to weak or strong correlations $(0 \leq r \leq 1)$ : $(A \leftrightarrow B) ;$

The method seems promising as it concerns Digital Humanities and Machine Learning. Many machine learning techniques make use of human beings to label the corpus to avoid to define the involved labels, at the risk of mistakes and ambiguity. Quantum semantics offers a different perspective on meaning, which can be useful to re-classify the corpus. For example, many hate speeches present strong anti-correlations between terms no matter of the width of the window. Furthermore, similar semantic profiles such as NH.WhW-B.2, H.WhW-B.3, NH.BW-Wh.4, H.BW-Wh.1 reveal a similar topic (black or white women) and show a sexist connotation, no matter how they have been labelled.

\subsection{Semantics and information}

As we wrote, the method we described applies to Semantics only in a narrow sense (see paragraph 3). Actually, the algorithm does not understand meaning: its task is not to translate texts; the algorithm provides information on meaning: on textual semantic structure, on some functions of the semantic system that produces the text (in our case: $a \vee b ; c \rightarrow d$ ) in the light of the semiotic square [9]. For example, the algorithm could be applied to a document written in a previously unknown language such as Minoan linear A script, or to an encrypted one. As long as we can distinguish its words, the algorithm would not decode the document, but it would provide information on what lexemes can be considered similar, implied or opposed as meaning is regarded, mining information from their co-occurence in the text and measuring the expectations related to some simple transformations operated on the coherent distributions of meaning - isotopies [10] - along the text.

\subsection{Future work}

In this paper we measured the correlation with reference to the single Pauli operator $\sigma_{x}$. To improve the method, we will measure the expectations of Pauli's operators $\sigma_{y}$ and $\sigma_{z}$ to get an alternative way to measure entanglement[18], to be compared to Bell inequalities used by Barros et al. [2]. On a similar line, the Born rule allows us to work on density matrices. Thus we hope to get further insights on the relation between Von Neumann information and meaning.

\section{References}

[1] (2018): The Online Hate Index. Available at http://www.adl.org/resources/reports/ the-online-hate-index 
[2] João Barros, Zeno Toffano, Youssef Meguebli \& Bich-Liên Doan (2014): Contextual Query Using Bell Tests. In Harald Atmanspacher, Emmanuel Haven, Kirsty Kitto \& Derek Raine, editors: Quantum Interaction, Springer Berlin Heidelberg, Berlin, Heidelberg, pp. 110-121, doi:10.1007/978-3-642-54943-4_10.

[3] Peter Bruza \& John Woods (2008): Quantum collapse in semantic space : interpreting natural language argumentation. In: Proceedings of the Second Quantum Interaction Symposium (QI-2008), College Publications, pp. 141-147.

[4] John F Clauser, Michael A Horne, Abner Shimony \& Richard A Holt (1969): Proposed experiment to test local hidden-variable theories. Physical review letters 23(15), p. 880, doi:10.1103/PhysRevLett.23.880.

[5] Gilles Deleuze \& Félix Guattari (1988): A thousand plateaus: Capitalism and schizophrenia. Bloomsbury Publishing.

[6] François Dubois \& Zeno Toffano (2017): Eigenlogic: A Quantum View for Multiple-Valued and Fuzzy Systems. In Jose Acacio de Barros, Bob Coecke \& Emmanuel Pothos, editors: Quantum Interaction, Springer International Publishing, Cham, pp. 239-251, doi:10.1007/978-3-642-54943-4_10

[7] Umberto Eco (2014): From the tree to the labyrinth. Harvard University Press, doi $10.4159 / 9780674728165$

[8] Francesco Galofaro, Zeno Toffano \& Bich-Liên Doan (2018): A quantum-based semiotic model for textual semantics. Kybernetes 47(2), pp. 307-320, doi:10.1108/K-05-2017-0187.

[9] A. J. Greimas \& François Rastier (1968): The Interaction of Semiotic Constraints. Yale French Studies (41), pp. 86-105, doi 10.2307/2929667. Available at http://www.jstor.org/stable/2929667.

[10] Algirdas Julien Greimas (1983): Structural semantics: An attempt at a method. University of Nebraska Press.

[11] Kevin Lund \& Curt Burgess (1996): Producing high-dimensional semantic spaces from lexical co-occurrence. Behavior Research Methods, Instruments, \& Computers 28(2), pp. 203-208, doi $10.3758 / \mathrm{BF} 03204766$.

[12] Massimo Melucci (2015): Introduction to information retrieval and quantum mechanics. Springer, Berlin, Heidelberg, doi: 10.1007/978-3-662-48313-8.

[13] Michael A Nielsen \& Isaac L Chuang (2004): Quantum Computation and Quantum Information (Cambridge Series on Information and the Natural Sciences). Cambridge university press.

[14] Jean Petitot (2004): Morphogenesis of meaning. P. Lang.

[15] François Rastier (2009): Sémantique interprétative. Presses universitaires de France, doi $10.3917 /$ puf.rast.2009.01

[16] Amit Singhal (2001): Modern Information Retrieval: A Brief Overview. IEEE Data Eng. Bull. 24(4), pp. 35-43. Available at http://sites.computer.org/debull/A01DEC-CD.pdf

[17] Dawei Song, Dawei Song, Peter Bruza \& Richard Cole (2004): Concept Learning and Information Inferencing on a High Dimensional Semantic Space. In: ACM SIGIR 2004 Workshop on Mathematical/Formal Methods in Information Retrieval (MF/IR2004). Sheffield, United Kingdom, 25-29 July 2004., doi 10.1 .1 .370 .4676

[18] Leonard Susskind \& Art Friedman (2014): Quantum mechanics: the theoretical minimum. Basic Books (AZ).

[19] Cornelis Joost Van Rijsbergen (2004): The geometry of information retrieval. Cambridge University Press, doi $10.1017 / \mathrm{CBO} 9780511543333$.

[20] Dmitry Zinoviev (2016): Data Science Essentials in Python: Collect-Organize-Explore-Predict-Value. Pragmatic Bookshelf. 